\title{
Calcutation of kaon matrix elements in quenched domain-wall QCD with DBW2 gauge action
}

\author{
J. Noaki ${ }^{\mathrm{a}}$ for RBC Collabolation* \\ ${ }^{a}$ RIKEN BNL Research Center, Brookhaven National Laboratory, Upton, NY 11973-5000, USA
}

\begin{abstract}
We give a progress report of our new $a^{-1} \approx 3 \mathrm{GeV}$ quenched calculation of kaon matrix elements with domainwall fermion and DBW2 gauge action. Our smaller lattice spacing allows us to address the effect of charmed quark on the lattice. We show preliminary results of $B_{K}$ renormalized non-perturbatively and $K \rightarrow \pi$ matrix elements.
\end{abstract}

\section{Introduction}

In order to treat the QCD effect in non-leptonic kaon decays non-perturbatively, it is important for the matrix elements of the local operators to be calculated on the lattice. A couple of years ago, CP-PACS and RBC Collaboration [12] calculated all of the matrix elements for the interaction of $K \rightarrow \pi \pi$ decay: $H_{W}=\sum_{i} C_{i}(\mu) Q_{i}$. Using the domain-wall fermion formalism to realize the chiral symmetry required in this calculation, they reported small and negative values of $\epsilon^{\prime} / \epsilon$ in conflict with the experimental result. In these works, however, there are several uncontrolled systematic errors coming from 1) the small, but non-zero breaking of chiral symmetry, 2) finite lattice spacing, 3) the perturbative treatment of the charmed quark in the matrix elements, 4) quenching effect, and 5) $K \rightarrow \pi \pi$ matrix elements obtained from $K \rightarrow \pi$ and $K \rightarrow 0$ by using lowest order chiral perturbation theory $[3$.

In order to examine the first three of above systematic errors extensively, we are performing a quenched simulation with domain-wall fermion and the DBW2 gauge action which improves the chiral symmetry on the lattice [4. The degree of chiral symmetry breaking is decreased by a factor 1/10 compared with the previous work of RBC Collaboration. In addition, the effect of the charmed quark on the lattice can be examined as

\footnotetext{
*We thank RIKEN, BNL and the U.S. DOE for providing
} the facilities essential for the completion of this work. well as the scaling violation.

In the rest of this article, we present the contents of the numerical simulation and report preliminary results of kaon B-parameter $B_{K}$ and the matrix elements which numerically dominate $\Delta I=1 / 2$ rule and $\epsilon^{\prime} / \epsilon$. Our dynamical simulation to study the quenching effect is reported in refs. [5].

\section{Numerical Simulation}

In Table. 1] simulation parameters used in this calculation and preliminary results of basic quantities are summarized. Our strategy of gauge generation with a rather fine lattice spacing and well-distributed topological charge is discussed in ref. [6]. Since quark mass $m_{f} a$ is introduced as a parameter of the boundary condition in the fifth dimension in domain-wall QCD, localization of chiral modes on both domain-walls in the fifth dimension tends to fail for a heavy quark mass. However, our small lattice spacing made the value of $m_{c} a$ acceptable as a domain-wall fermion: $m_{c} a \simeq 0.45$. We observed that, around this value, the shape of the wave function in the fifth dimension was qualitatively similar to that with much smaller $m_{f}$. The small value of residual quark mass $m_{\text {res }} \lesssim 0.3 \mathrm{MeV}$ demonstrates the good chiral symmetry. Therefore, it is expected that at least for those operators that do not mix with lower dimensional ones, finite $L_{s}$ causes negligible chiral symmetry breaking effects. 


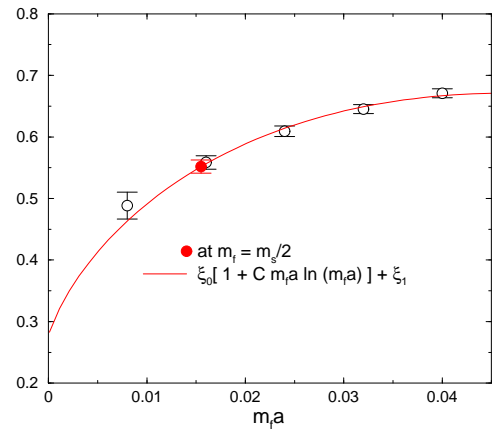

Figure 1. Lattice value of $B_{K}$ as a function of $m_{f} a$.
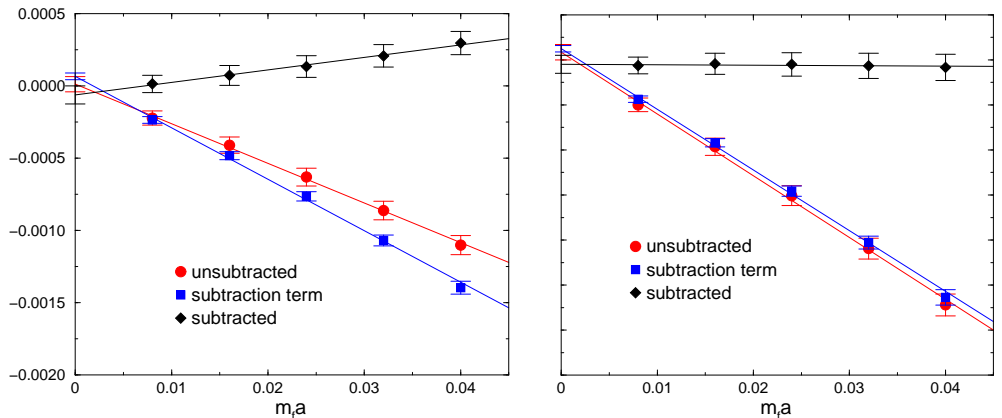

Figure 2. $K \rightarrow \pi$ matrix elements of $Q_{2}^{(0)}$ (left) and $Q_{2 c}$ (right, with $\left.m_{c} a=0.40\right)$ as a function of $m_{f} a$. Linear fit was used.

\section{Kaon B-parameter $B_{K}$}

$B_{K}$ on the lattice can be obtained as a ratio of the matrix elements of $Q_{\Delta S=2}=\left[\bar{s} \gamma_{\mu}\left(1-\gamma_{5}\right) d\right]^{2}$ and axial current $A_{4}$. Our result is shown in Fig. [1] as a function of $m_{f} a$. The fit function used is $B_{K}=\xi_{0}\left[1+C m_{f} a \ln \left(m_{f} a\right)\right]+\xi_{1} m_{f} a$ with the coefficient $C$ taken from analytic result [7. In this chiral fit, $\chi^{2} /$ dof $=0.76$ is obtained.

Taking only statistical errors into account, our preliminary results are summarized as

$$
\begin{aligned}
& B_{K}^{\text {latt }}\left(m_{f} a=0.0155\right)=0.552(11), \\
& B_{K}^{\mathrm{RI}-\mathrm{MOM}}(\mu=2 \mathrm{GeV})=0.542(11), \\
& B_{K}^{\overline{\mathrm{MS}}, \mathrm{NDR}}(\mu=2 \mathrm{GeV})=0.549(11), \\
& \hat{B}_{K}=0.764(15) .
\end{aligned}
$$

Table 1

Simulation parameters and preliminary results of basic quantities.

\begin{tabular}{rl}
\hline size: & $24^{3} \times 48$ \\
DBW2: & $\beta=1.22$, \\
\#sweeps & $5,000\left(B_{K}\right), 10,000(K \rightarrow \pi$, meson $)$ \\
DWF: & $M_{5}=1.65, L_{s}=10$ \\
$m_{f} a$ & $0.008-0.040$, in step of 0.008 \\
$m_{c} a$ & $0.08,0.12,0.16,0.20,0.30,0.40,0.50$ \\
$\#$ configs. & $77\left(B_{K}\right), 23(\mathrm{NPR}), 42\left(m_{\mathrm{res}}\right)$, \\
& $50(K \rightarrow \pi$, meson $)$ \\
\hline$a^{-1}$ & $2.86(9) \mathrm{GeV}\left(m_{f} a=0\right.$, from $\rho$-meson $)$ \\
$m_{\mathrm{res}} a$ & $9.73(4) \cdot 10^{-5}\left(m_{f} a=0\right)$ \\
\hline
\end{tabular}

The lattice value $B_{K}^{\text {latt }}$ is quoted at $m_{f}=m_{s} / 2$ (filled symbol in Fig. (1). By a non-perturbative renormalization procedure following 9, we obtained renormalization factor $Z_{B_{K}}^{\mathrm{RI}-\mathrm{MOM}}(\mu=$ $2 \mathrm{GeV})=0.9816(79)$. In this step, we also estimated the degree of the mixing of the operators with wrong chirality. As expected by the small value of $m_{\text {res }}$, all elements of the amputated fourpoint function in the chirality basis were observed to be less than $0.2 \%$ of the $(V V+A A, V V+A A)$ element which corresponds to $Z_{B_{K}}$. Though we shifted $B_{K}^{\mathrm{RI}-\mathrm{MOM}}$ to $B_{K}^{\overline{\mathrm{MS}}}[10$ and the renormalization group invariant (RGI) value $\hat{B}_{K}$ 11] with $N_{f}=0$, results will change less than $1 \%$ even with $N_{f}=3$. Our result of $B_{K}^{\overline{\mathrm{MS}}}(\mu=2 \mathrm{GeV})$ is consistent with CP-PACS 8 ] $\left(a^{-1} \simeq 2.9 \mathrm{GeV}\right.$, $\left.24^{3} \times 60 \times 16\right)$ and the previous work of RBC [2].

\section{4. $K \rightarrow \pi$ matrix elements}

At the lowest order of chiral perturbation theory, $K \rightarrow \pi \pi$ matrix elements are proportional to $K \rightarrow \pi$ matrix elements calculated on the lattice 3 . For $i=1-6,9$ and 10 , these matrix elements are related as

$$
\begin{aligned}
& \left\langle\pi^{+} \pi^{-}\left|Q_{i}^{(I)}\right| K^{0}\right\rangle= \\
& \frac{m_{K}^{2}-m_{\pi}^{2}}{\sqrt{2} f}\left[\frac{\left\langle\pi^{+}\left|Q_{i}^{(I)}\right| K^{+}\right\rangle_{\mathrm{sub}}}{m_{\mathrm{PS}}^{2}}+\mathcal{O}\left(m_{\mathrm{PS}}^{2}\right)\right]
\end{aligned}
$$



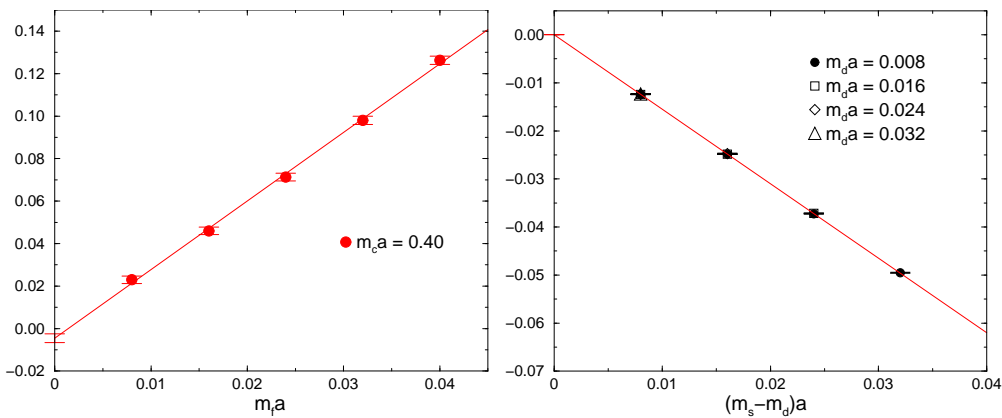

Figure 3. Unsubtracted $K \rightarrow \pi$ matrix element of $Q_{6}^{(0)}$ and a ratio of $K \rightarrow 0$ matrix elements of $Q_{6}^{(0)}$ and $\bar{s} \gamma_{5} d$. Linear fit was used for both cases.

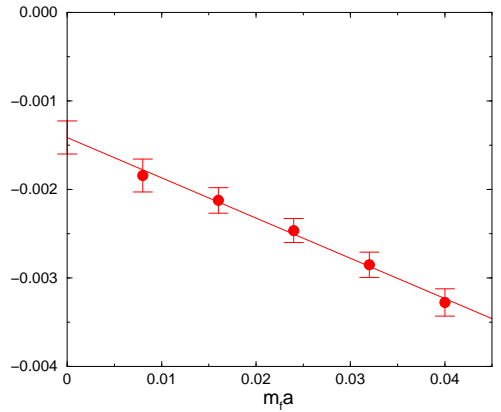

Figure $4 . K \rightarrow \pi$ matrix element of $Q_{8}^{(2)}$ as a function of $m_{f} a$ with its liniar fit. where $Q_{i}^{(I=0,2)}$ is the contribution to the final state with $I=0,2$. Only for the case of $\Delta I=$ $1 / 2$, or $I=0$, subtraction is needed to resolve mixing with a lower dimensional operator:

$$
\begin{aligned}
&\left\langle\pi^{+}\left|Q_{i}^{(0)}\right| K^{+}\right\rangle_{\text {sub }}=\left\langle\pi^{+}\left|Q_{i}^{(0)}-\alpha_{i} Q_{\mathrm{sub}}\right| K^{+}\right\rangle \\
& Q_{\mathrm{sub}} \equiv\left(m_{s}+m_{d}\right) \bar{s} d-\left(m_{s}-m_{d}\right) \bar{s} \gamma_{5} d \\
& \alpha_{i}=\left\langle 0\left|Q_{i}^{(0)}\right| K^{0}\right\rangle /\left\langle 0\left|Q_{\mathrm{sub}}\right| K^{0}\right\rangle
\end{aligned}
$$

In Fig. 2] $K \rightarrow \pi$ matrix elements of $Q_{2}^{(0)}$ (left) and $Q_{2 c}=(\bar{s} u)_{L}(\bar{u} d)_{L}$ (right) are presented before and after the subtraction of (2). In particular, one finds that the slope of the subtracted matrix element of $Q_{2 c}$ is much smaller than that of $Q_{2}^{(0)}$, which might mean a minor contribution of $\left\langle\pi \pi\left|Q_{2 c}\right| K\right\rangle$ to $\Delta I=1 / 2$ rule.

Fig. [3 shows $\left\langle\pi^{+}\left|Q_{6}^{(0)}\right| K^{+}\right\rangle$without the subtraction (left) and $\left\langle 0\left|Q_{6}^{(0)}\right| K^{0}\right\rangle /\left\langle 0\left|\bar{s} \gamma_{5} d\right| K^{0}\right\rangle$ as a function of $m_{s} a-m_{d} a$ (right) whose slope should be $\alpha_{6}$. Though these are an example with $m_{c} a=0.40$, their dependence on $m_{c} a$ is not visible, so far. While the slopes of both quantities are determined within the error of $2 \%$ and $0.2 \%$ respectively, $K \rightarrow \pi \pi$ matrix element which is obtained as a combination of them has the error more than $200 \%$ due to a severe subtraction. Therefore, before we can quote result for $\left\langle\pi^{+} \pi^{-}\left|Q_{6}^{(0)}\right| K^{0}\right\rangle$, we need to significantly improve our statistics.
For $i=7$ and $8, K \rightarrow \pi \pi$ and $K \rightarrow \pi$ matrix elements are related as

$$
\begin{aligned}
\left\langle\pi^{+} \pi^{-}\left|Q_{i}^{(I)}\right| K^{0}\right\rangle= & -\frac{1}{\sqrt{2} f}\left\langle\pi^{+}\left|Q_{i}^{(I)}\right| K^{+}\right\rangle \\
& +\mathcal{O}\left(m_{\mathrm{PS}}^{2}\right) .
\end{aligned}
$$

We show $\left\langle\pi^{+}\left|Q_{8}^{(2)}\right| K^{+}\right\rangle$in Fig. 4 and observe its expected shape and intercept roughly consistent with previous works 12.

\section{REFERENCES}

1. J. Noaki et al. (CP-PACS Collaboration), Phys. Rev. D68 (2003) 014501.

2. T. Blum et al. (RBC Collaboration), hep-lat/0110075, to appear in Phys. Rev. D.

3. C. Bernard et al., Phys. Rev.D32(1985) 2343.

4. Y. Aoki et al. (RBC Collaboration), hep-lat/0211023

5. See contributions of C. Dawson, T. Izubuchi and R. Mawhinney to these proceedings.

6. J. Noaki for RBC Collaboration, Nucl. Phys. B (Proc. Suppl.) 119 (2003) 362.

7. Sharpe, S., Phys. Rev. D46 (1992) 3146.

8. A. Ali Khan et al. (CP-PACS Collaboration), Phys. Rev. D64 (2001) 114506.

9. G. Martinelli et al., Nucl. Phys. B445 (1995) 81; T. Blum et al. (RBC Collaboration), Rhys. Rev. D 66 (2002) 014504.

10. M. Ciuchini et al., Z. Phys. C68 (1995) 239.

11. M. Ciuchini et al., Nucl. Phys. B523 (1998) 501. 